\title{
Analysis of the tight skin $(T s k 1 /+)$ mouse as a model for testing antifibrotic agents
}

\author{
Ruth M Baxter, Thomas P Crowell, Margaret E McCrann, Erica M Frew and \\ Humphrey Gardner \\ Departments of Research Pathology and Molecular Discovery, Biogen Idec, 14 Cambridge Center, \\ Cambridge, MA, USA
}

\begin{abstract}
The tight skin $1\left(\mathrm{~B} 6 . \mathrm{CgFbn} 1^{T s k}+I+P I d n^{p a} / \mathrm{J}\right.$, henceforth referred to as $\left.T s k 1 /+\right)$ mouse was first described as a spontaneously occurring mutant that resulted in hyperplasia of the subcutaneous loose connective tissue, and has subsequently been proposed to be a model of the human fibrotic disorder scleroderma. We have investigated the Tsk1/+ mouse as a model system for testing the efficacy of antifibrotic agents against skin fibrosis. We find that the tightness of the skin at the scruff of the neck leads to a measurably thicker skin pinch, but we suggest that this is due to hyperplasia of the subdermal loose connective tissue, which results in increased tethering of the skin to the underlying muscle layers. In contrast to previously published data, we do not find a significant difference in the dermal thickness or collagen content of the Tsk1/+ mouse skin compared with wild-type controls. In addition, expression profiling of $T s k 1 /+$ mouse skin indicated that there are very few changes in gene expression, and that there is no evidence for upregulation of the transforming growth factor $\beta$ signaling axis. Therefore, we conclude that this model is not suitable for testing the effect of antifibrotic agents on the dermis, and that changes potentially related to scleroderma may be confined to subdermal connective tissue.
\end{abstract}

Laboratory Investigation (2005) 85, 1199-1209. doi:10.1038/labinvest.3700331; published online 15 August 2005

Keywords: fibrillin; fibrosis; scleroderma; tight skin mouse; transcript profiling

Fibrillin-1, a major component of microfibrils, ${ }^{1,2}$ has been implicated in the development of scleroderma. The chromosomal region containing Fbn1 is strongly associated with the development of scleroderma in the Choctaw Native Americans, who have a much higher incidence of scleroderma than the general population, ${ }^{3-5}$ and increased fibrillin-1 has been observed in scleroderma skin lesions. ${ }^{6}$ Antifibrillin-1 antibodies have been found in patients with scleroderma, ${ }^{7,8}$ and microfibrils produced by fibroblasts derived from scleroderma patients appear to be unstable. ${ }^{9}$

Fibrillin-1 has a structural role in connective tissue as a component of elastic fibers. It is also hypothesized to have a role in controlling the biologically available levels of the profibrotic cytokine, transforming growth factor $\beta$ (TGF $\beta),{ }^{10,11}$ through binding to the latent TGF $\beta$ binding proteins

Correspondence: Dr H Gardner, MD, Department of Research Pathology, Biogen Idec, 14 Cambridge Center, Cambridge, MA 02142, USA.

E-mail: humphrey.gardner@biogenidec.com

Received 16 May 2005; revised 30 June 2005; accepted 5 July 2005; published online 15 August 2005
(LTBP) 1 and $4 .^{10,12,13}$ TGF $\beta$ has long been implicated in the pathogenesis of scleroderma ${ }^{14,15}$ and other fibrotic disorders reviewed in Leask and Abraham. ${ }^{16}$

We are interested in developing pharmacologic inhibitors of fibrosis that may be efficacious in diseases such as scleroderma. The tight skin 1 $(T s k 1 /+)$ mouse occurred as a spontaneous mutation as the result of a partial in-frame duplication in fibrillin- $1,{ }^{17}$ and the initial description of the phenotype was one of hyperplasia of the subcutaneous loose connective tissue. ${ }^{18}$ Subsequently, investigators showed that the Tsk1/+ mouse developed thickened dermis, ${ }^{19}$ and that the structure of the dermis is abnormal. ${ }^{20}$ The Tsk1/+ mouse was proposed to be a model of scleroderma, ${ }^{21}$ and it has been demonstrated that there is a correlation between skin thickness, hydroxy-proline content of the dermis, soluble collagen content and presence of autoantibodies in the Tsk1/+ mouse. ${ }^{22}$ The Tsk1/+ mouse has now become a widely used model for scleroderma and skin fibrosis. ${ }^{23-25}$ In addition to many papers showing hyperplasia and increased collagen content of the dermis, ${ }^{25-30}$ it has been suggested that activation of the TGF $\beta$ signaling axis is involved in the development of the Tsk1/+ 
phenotype. ${ }^{28,31}$ Owing to the many publications showing effects of various gene deletions and pharmacologic agents on the thickness of the dermis in the $T s k 1 /+$ mouse, ${ }^{27-29,32,33}$ we decided to use this model to test the effects of potential antifibrotic agents in skin fibrosis.

Here, we report that we found consistent changes in skin parameter measurements in vivo, as well as emphysematous changes in the lungs. However, we did not find thickened dermis or increased dermal collagen content in the Tsk1/+ mouse compared with age- and sex-matched wild-type controls. Consistent with our expectations, we did find siteto-site variations in dermal thickness as well as marked differences between the sexes. We conclude that the histologic assessment of dermal thickness cannot be used to assay the efficacy of antifibrotics in Tsk1/ + mice.

\section{Materials and methods}

\section{Mice}

B6.Cg-Fbn $1^{T s k}+/+P l d n^{p a} / \mathrm{J}(T s k 1 /+)$ mice were obtained from The Jackson Laboratory, and maintained on the C57BL/6 background harboring the pallid mutation. Age-matched male Tsk1/ + and $\mathrm{pa} / \mathrm{pa}$ (wild-type) controls were used in all experiments. All animal experiments were approved by the institutional animal care and use committee.

\section{Histological Analysis}

For comparisons of dermal thickness, skin was excised from directly over the shoulder blades and spread onto a piece of filter paper prior to fixation. Lungs were inflated with $1 \mathrm{ml}$ of $10 \%$ neutralbuffered formalin (NBF) before removal. All tissue samples were fixed in 10\% NBF and processed in paraffin. Paraffin sections $(5 \mu \mathrm{m})$ were prepared, heated for $20 \mathrm{~min}$ at $65^{\circ} \mathrm{C}$, deparaffinized in xylene and hydrated through graded alcohols to distilled water. For H\&E staining, sections were stained with Mayer's hematoxylin (Sigma) for $8 \mathrm{~min}$, rinsed in water, counterstained with Eosin-Phloxine solution (0.1\% Eosin Y, 0.01\% Phloxine B in 95\% ETOH) for $1 \mathrm{~min}$, dehydrated through graded alcohols and cleared in xylene. For detection of collagen, sections were stained with Picrosirius Red solution (Sirius Red F3BA, $0.1 \%$ in saturated picric acid, prepared in the laboratory) for $30 \mathrm{~min}$, rinsed in $0.01 \mathrm{~N} \mathrm{HCl}$ for $2 \mathrm{~min}$, dehydrated through graded alcohols and cleared in xylene. Coverslips were applied using Permount. Image analysis to quantify Picrosirius red-positive areas was carried out using Colorcounter software. Statistical significance of differences between Tsk1/+ and wild-type mice for inlife and histological measurements was assessed using Student's $t$-test.

\section{Adult Mouse Primary Dermal Fibroblasts}

The area of skin to be used was shaved and treated with hair-removing lotion to remove the hair completely. Excised skin was spread dermis side down in a sterile tissue culture dish and incubated overnight with $0.25 \%$ trypsin at $4^{\circ} \mathrm{C}$. Following removal of the epidermis, the dermis was chopped into small pieces and incubated with $0.25 \%$ Collagenase Type I (Worthington) in DMEM at $37^{\circ} \mathrm{C}$ for $4 \mathrm{~h}$, then dissociated into a cellular suspension. Cells were washed twice to remove collagenase then cultured in DMEM plus 10\% FBS until confluent. For treatment with TGF $\beta$, confluent cultures were incubated in DMEM plus 5\% FBS supplemented with $1 \mathrm{ng} / \mathrm{ml}$ TGF $\beta$ (R\&D Systems).

\section{Detection of Fibrillin}

Confluent cultures of primary fibroblasts in $60 \mathrm{~mm}$ dishes were incubated with serum-free DMEM for $72 \mathrm{~h}$ prior to collection of medium. Proteins were precipitated from $100 \mu \mathrm{l}$ of conditioned medium by the addition of $10 \%$ (final concentration) TCA, separated on a $3-8 \%$ protein gel (Novex) and transferred to PVDF membranes. Blots were probed with a polyclonal antifibrillin antibody pAb9543 raised against the $\mathrm{NH}_{2}$-terminus of the protein (kindly provided by Professor Lynn Sakai), followed by detection with anti-rabbit-HRP-conjugated secondary antibody (Pharmacia) and ECL reagent (Pharmacia).

\section{Caliper Measurements}

A digital spring-loaded micrometer was purchased from Mitutoyo. To measure the thickness of the skin pinch at the scruff of the neck, the skin of unshaved mice was pinched manually and the micrometer applied to the skin pinch. For ear thickness measurements, the micrometer was applied to the tip of the ear pinna so that the entire area of the caliper head was in contact with the skin.

\section{Preparation of Total RNA from Skin}

The area of skin to be taken was first shaved and swabbed with $70 \%$ ethanol. Skin samples were immediately frozen in liquid nitrogen then ground to a fine powder before being homogenized in $1 \mathrm{ml}$ of TRI reagent (Sigma). A $20 \%$ volume of $\mathrm{CHCl}_{3}$ was added to the homogenate and samples vigorously mixed. Following centrifugation, the aqueous upper layer was transferred to a clean tube and an equal volume of $70 \%$ ethanol added. Total RNA was purified using Nucleospin columns (BD Biosciences), according to the manufacturer's directions. 


\section{Real-Time PCR}

cDNA was prepared from $0.5 \mu \mathrm{g}$ total RNA using PrePrimed SingleShots with random hexamer primers (BD Biosciences). Levels of Col1a2 were analyzed by real-time PCR using Tsg DNA polymerase and sybr green dye (Lamda Biotech) fluorescence to detect product. Primer sequences for Col1a2 are as follows: forward, $5^{\prime}$ GGCAAGACA ATCATTGAA; and reverse, 5' GGTTGAGTTCACT TATTTGAA. Each reaction was carried out in duplicate, using $5 \mu \mathrm{l}$ of a 1:1000 dilution of the cDNA generated in the reverse transcriptase reaction.

\section{Probe Labeling, Hybridization and Scanning}

Sample labeling, hybridization and staining were carried out according to the Eukaryotic Target Preparation protocol in the Affymetrix Technical Manual (701021 rev. 4) for Genechip ${ }^{\circledR}$ Expression Analysis (Affymetrix). In summary, $5 \mu \mathrm{g}$ of purified total RNA was used in a $20 \mu \mathrm{l}$ first-strand reaction with $200 \mathrm{U}$ SuperScript II (Invitrogen) and $0.5 \mu \mathrm{g}$ (dT)-T7 primer (5'-GGCCAGTGAATTGTAATAC GACTCACTATAGGGAGGCGG $(\mathrm{T})_{24}$ ] in $1 \times$ firststrand buffer (Invitrogen) with a $42^{\circ} \mathrm{C}$ incubation for $1 \mathrm{~h}$. Second-strand synthesis was carried out by the addition of $40 \mathrm{U}$ Escherichia coli DNA polymerase, $2 \mathrm{U}$ E. coli RNase $\mathrm{H}$ and $10 \mathrm{U}$ E. coli DNA ligase in $1 \times$ second-strand buffer (Invitrogen) followed by incubation at $16^{\circ} \mathrm{C}$ for $2 \mathrm{~h}$. The second-strand synthesis reaction was purified using the Genechip ${ }^{\circledR}$ Sample Cleanup Module according to the manufacturer's protocol (Affymetrix). The purified cDNA was amplified using BioArray HighYield RNA transcript labeling kit (T7) (Enzo Life Sciences) according to the manufacturer's protocol to produce biotin labeled cRNA. Mouse Genome MOE430A GeneChip $^{\circledR}$ probe arrays were prehybridized in a GeneChip $^{\circledR}$ Hybridization Oven 640 (Affymetrix) according to the manufacturer's protocol. Labeled cRNA $(15 \mu \mathrm{g})$ was fragmented in $30 \mu \mathrm{l} 1 \times$ fragmentation buffer at $94^{\circ} \mathrm{C}$ for $35 \mathrm{~min}$. The fragmented labeled cRNA was resuspended in $300 \mu \mathrm{l} 1 \times$ hybridization buffer containing $100 \mathrm{mM}$ MES, $1 \mathrm{M}$ $\left[\mathrm{Na}^{+}\right.$], $20 \mathrm{mM}$ EDTA, 0.01\% Tween-20, $0.5 \mathrm{mg} / \mathrm{ml}$ aceylated BSA, $0.1 \mathrm{mg} / \mathrm{ml}$ herring sperm DNA, control oligo-B2 and control transcripts bioB $1.5 \mathrm{pM}$, bioC $5 \mathrm{pM}$, bioD $25 \mathrm{pM}$ and cre $100 \mathrm{pM}$, and hybridized to Genechip ${ }^{\circledR}$ probe arrays according to the manufacturer's protocol (Affymetrix). The hybridized GeneChip ${ }^{\circledR}$ probe arrays were washed and stained according to the Affymetrix protocol for antibody amplification stain for eukaryotic targets using R-Phycoerythrin Streptavidin (Molecular Probes), goat IgG (Sigma) and biotinylated anti-streptavidin (Vector Laboratories) on the GeneChip $^{\circledR}$ Fluidics Station 450 (Affymetrix). The GeneChip $^{\circledR}$ probe arrays were scanned using GeneChip $^{\circledR}$ Scanner 3000.

\section{Gene Profiling Data Analysis}

Analyses were performed using BRB ArrayTools developed by Dr Richard Simon and Amy Peng Lam.

\section{Immunohistochemistry}

Paraffin sections $(5 \mu \mathrm{m})$ were prepared, heated for $20 \mathrm{~min}$ at $65^{\circ} \mathrm{C}$, deparaffinized in xylene and hydrated through graded alcohols to distilled water. Antigens were unmasked by boiling in $10 \mathrm{mM}$ citrate buffer, and samples incubated with antiphosphor-Smad 2 antibodies (Cell Signaling Technology). Staining was detected using biotinylated anti-rabbit secondary antibodies followed by ABC reagent (Vector laboratories) according to the manufacturer's directions.

\section{Results}

\section{Expression of Mutant Fibrillin}

The Tsk1/ + phenotype is the result of a partial inframe duplication of the Fbn 1 gene, which results in the production of a fibrillin protein that is larger than the wild-type protein. ${ }^{17}$ To ensure that the mice we were using were producing the mutant protein, Western blots were performed on conditioned medium from primary fibroblasts derived from wild-type and $T s k 1 /+$ mice, using a polyclonal antifibrillin antibody that recognizes both wildtype and mutant fibrillin. ${ }^{34}$ Figure 1 shows that, as expected, fibroblasts derived from Tsk1/ + mice (designated by skin color and increased skin tightness at the scruff of the neck) showed equal expression of a higher molecular weight band of fibrillin-1 in addition to a faster migrating form, while fibroblasts derived from wild-type mice showed only the faster migrating form.

\section{In-Life Measurements of Skin Thickness}

We used a spring-loaded digital micrometer to ensure that measurements were not compromised by variations in operator pressure using a manual caliper. We measured the thickness of the skin fold at the scruff of the neck of mice at different ages and found that $T s k 1 /+$ mice had measurably greater skin-fold thickness than the wild-type counterparts, but a much higher variance than the measurement of the wild-type mice (Figure 2a). We also tested the thickness of the ear pinna, and again found that we could see an appreciable difference between wildtype and $T s k 1 /+$, although in this case the Tsk1/+ was thinner than the wild type (Figure 2b). While the latter measurement is easy to perform and showed remarkable consistency with genotype, its dynamic range would be too small to discern drug effects. 


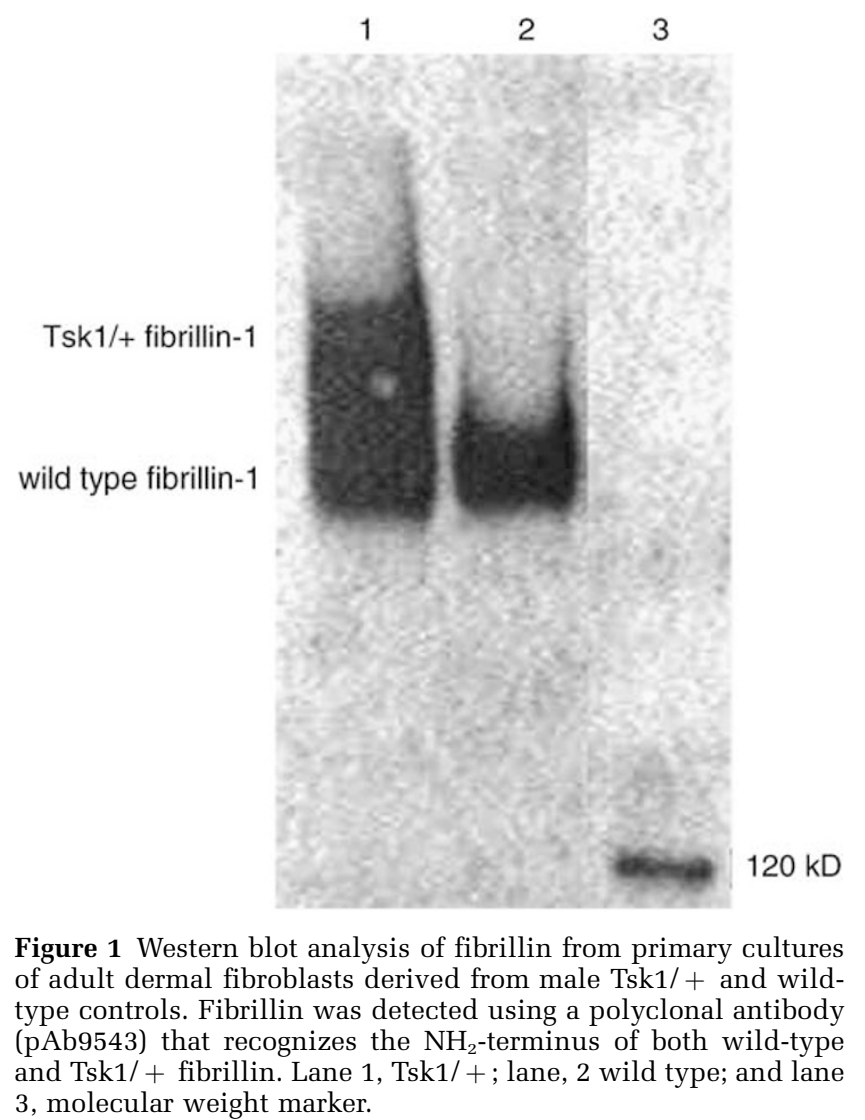

3 , molecular weight marker.

\section{Measurement of Dermal Thickness and Collagen Content}

Many reports have indicated that the dermis of the Tsk1/ + mouse is significantly thicker than that of wild-type mice, and that it contains more collagen. We examined the histology of dorsal skin taken from directly over the scapular region of male Tsk1/+ and age-matched wild-type controls. From examination of H\&E staining, we did not observe increased dermal thickness in Tsk1/ + compared to controls at any of the time points taken (Figure 3a shows representative sections of mice aged 12 and 16 weeks). However, consistent with previous findings, we did find increased subcutaneous tissue in the Tsk1/ + samples. ${ }^{18,35}$ To quantify the thickness of the dermis, the distance from the dermal/epidermal junction to the subcutaneous fat layer was measured at five positions along the length of each section (the lower limit of the dermis is indicated by dotted lines in Figure 3a). This analysis clearly shows that while there was considerable variance in dermal thickness between samples, there was no significant difference between wild-type and Tsk1I+ mouse skin (Figure 3b).

The amount of collagen in the dermis was assessed by quantification of Picrosirius red staining of skin. Figure 4a shows representative pictures of skin sections from male mice aged 12 and 16 weeks, and Figure $4 \mathrm{~b}$ shows total Sirius-positive pixels per
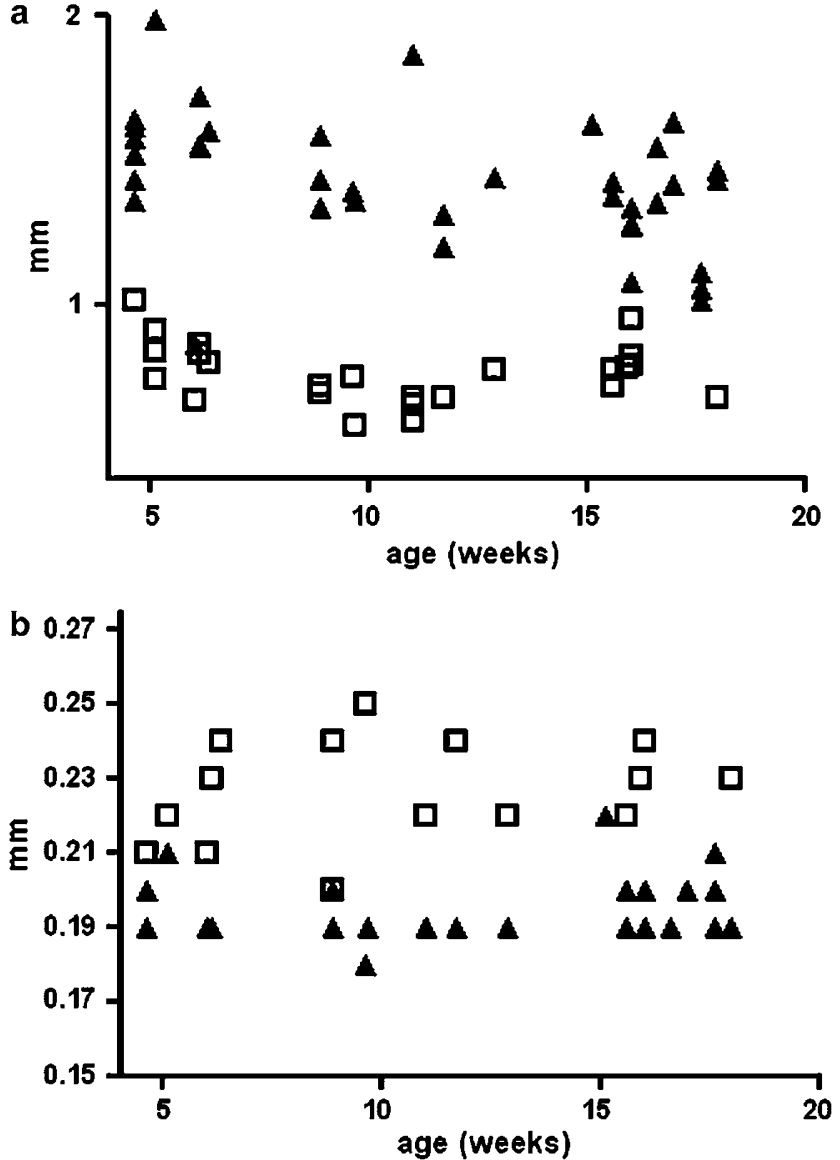

Figure 2 In vivo skin measurements. (a) Digital micrometer measurements of the skin-fold thickness of male Tsk1/ + and wild-type mice as a function of age. (b) Measurement of the thickness of the ear pinna as a function of age. Solid triangles represent Tsk1/ + and open squares represent wild type.

section for six wild-type and six Tsk1/+ mice, showing no increase in the amount of collagen in the Tsk1/+ skin. We also examined the expression of collagen at the level of transcription by real-time PCR using primers designed against mouse Col1a2. We did not find any difference in the amount of collagen mRNA expression between wild-type and Tsk1/ + skin at 6 weeks of age, nor in older mice aged 12-16 weeks (Figure 4c). To verify that we could see changes in Col1a2 expression using this assay, we measured the amount of Col1a2 in RNA derived from mouse dermal fibroblasts treated with TGF $\beta$ or left untreated. Figure $4 \mathrm{~d}$ shows that in this system, we detected a two-fold increase of Col1a2 upon TGF $\beta$ treatment of dermal fibroblasts.

\section{Lung Phenotype}

In addition to the skin changes, Tsk1/+ mice have also been reported to develop an emphysemalike condition. Examination of H\&E-stained sections of wild-type and Tsk1/ + lungs shows 

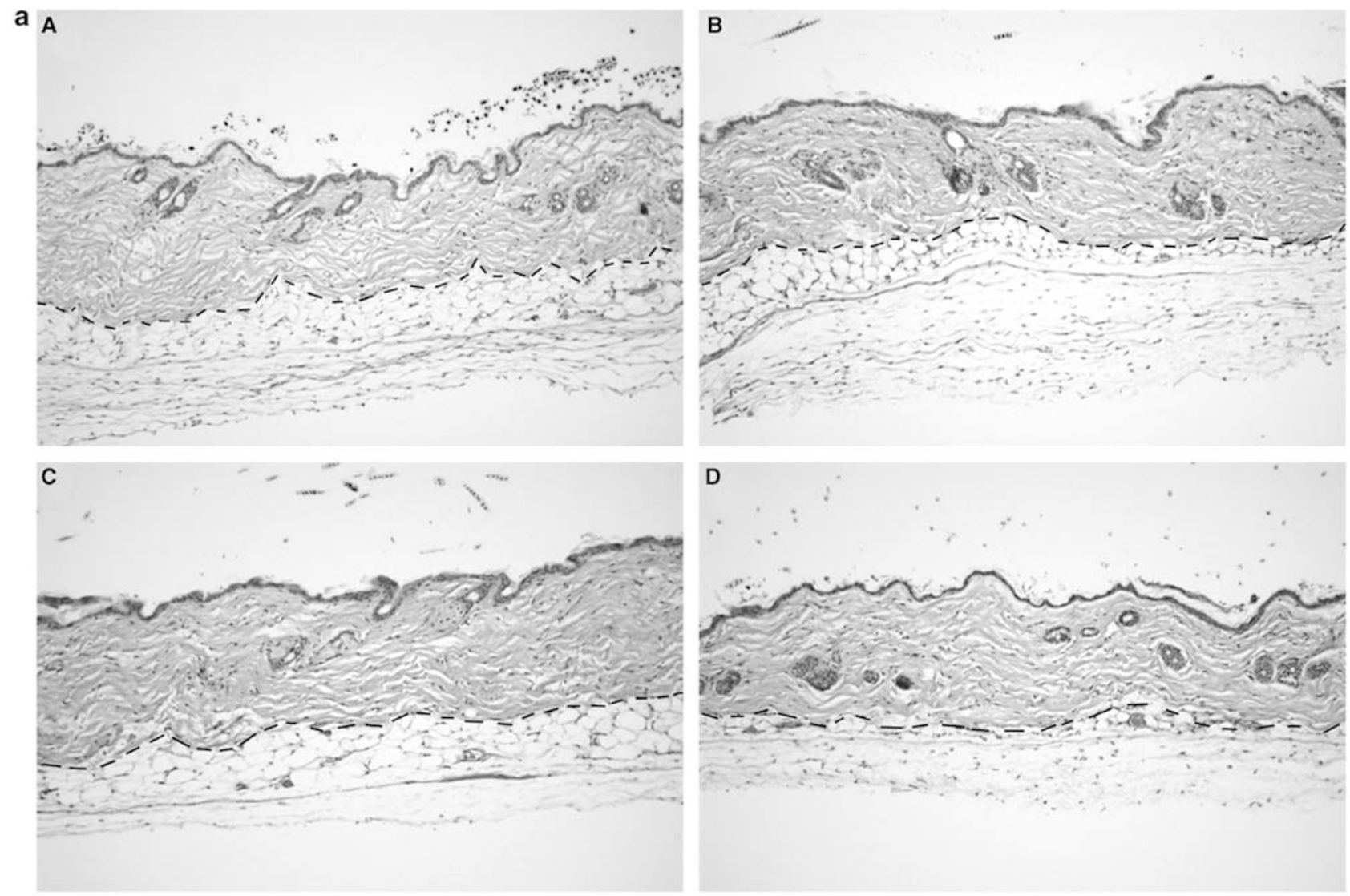

b

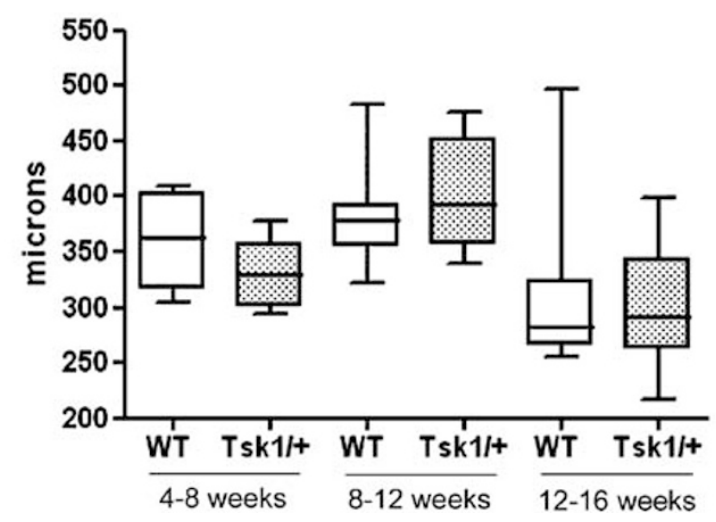

Figure 3 Histological analysis of Tsk1/ + and wild-type skin. (a) H\&E-stained skin sections from male Tsk1/ + (panels A and B) and wild type (panels C and D) age 12 weeks (panels A and C) and 16 weeks (panels B and D), pictures taken at $\times 10$ magnification. (b) Mean dermal thickness. The thickness of the dermis was determined by measuring the distance from the dermal/epidermal junction to the base of the dermis (indicated by dotted lines) at five positions along the length of each section. The plots show the median value, the 25th to 75th- percentile range and the maximum and minimum values for each group; $4-8$ weeks wild type $n=4, T s k 1 /+n=7 ; 8-12$ weeks wild type $n=8, T s k 1 /+n=9$; and 12-16 weeks wild type $n=10$, Tsk1 $+n=11$.

that the animals from our Tsk1/ + colony have greatly increased alveolar spaces compared with wild-type controls (Figure 5), consistent with published observations.

\section{Gene Expression Analysis}

To better understand the changes in Tsk1/ + skin, we performed transcript analysis on RNA derived 

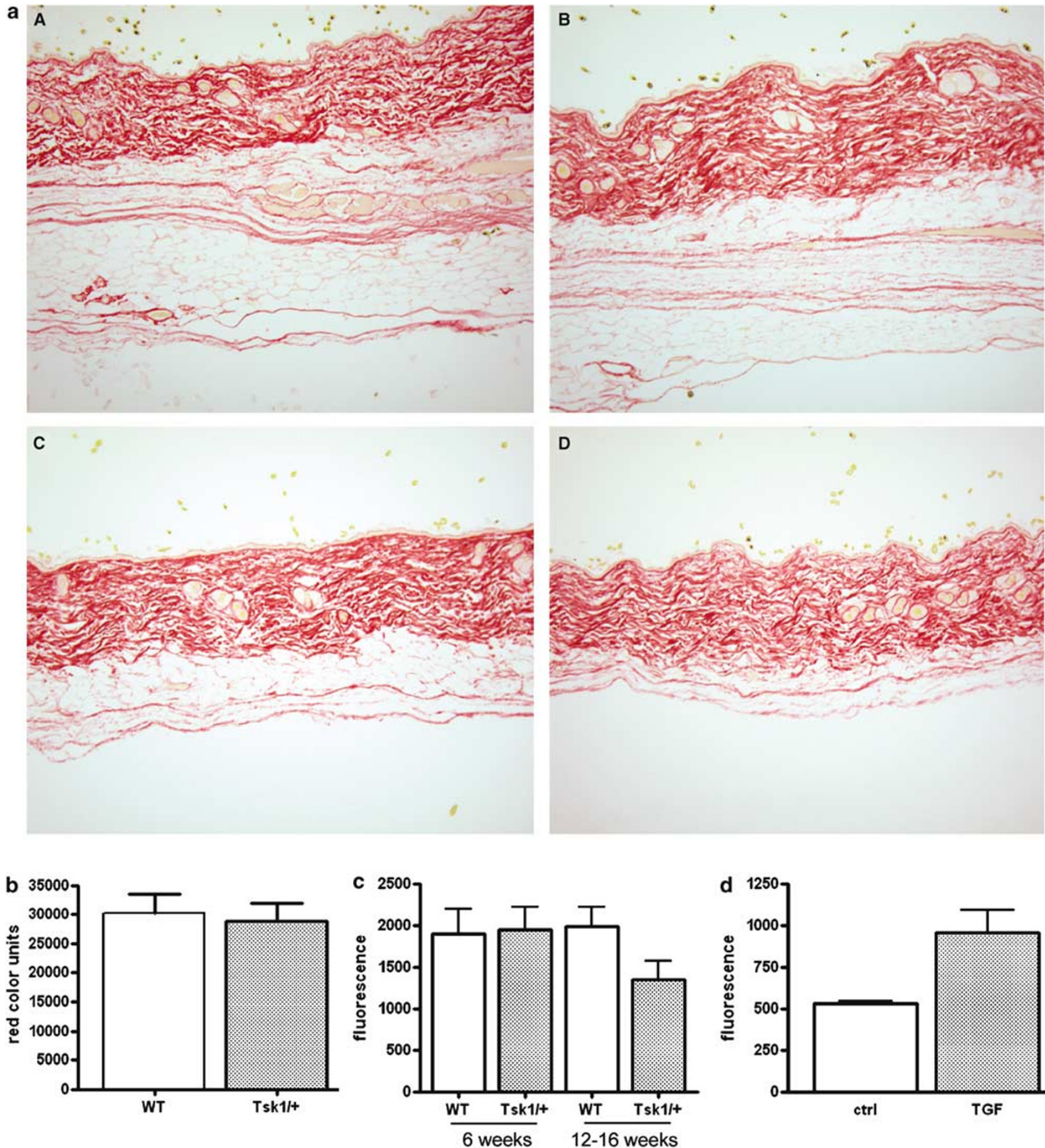

Figure 4 Analysis of collagen content of the dermis. (a) Picrosirius Red-stained skin sections from male Tsk1/ + (panels A and B) and wild-type (panels C and D) skin section at 12 weeks (panels $A$ and C) and 16 weeks (panels B and D) pictures taken at $\times 10$ magnification. (b) Total Picrosirius Red-positive area in skin sections of wild type $(n=6)$ and Tsk1/ $+(n=6)$. (c) Real-time PCR analysis of Col1a2 mRNA levels in 6-week-old wild type $(n=3)$ and Tsk1/ $+(n=3)$, and 12- to 16-week-old wild type $(n=3)$ and Tsk1/ $+(n=3)$. (d) Analysis of Col1a2 levels by real-time PCR in mouse dermal fibroblasts treated with TGF $\beta(1 \mathrm{ng} / \mathrm{ml})$.

that none of them were upregulated in the Tsk1/+ skin compared with controls. Pathways involved in the activation of TGF $\beta$, such as thrombospondin 1, and were also unchanged. Figure 6a shows a brief list of genes with expression levels indicated by a heat map in which green indicates lower expression and red higher expression. While there is variance in the expression levels of individual genes, there is no overall difference in expression between wildtype and Tsk1/ + samples. 

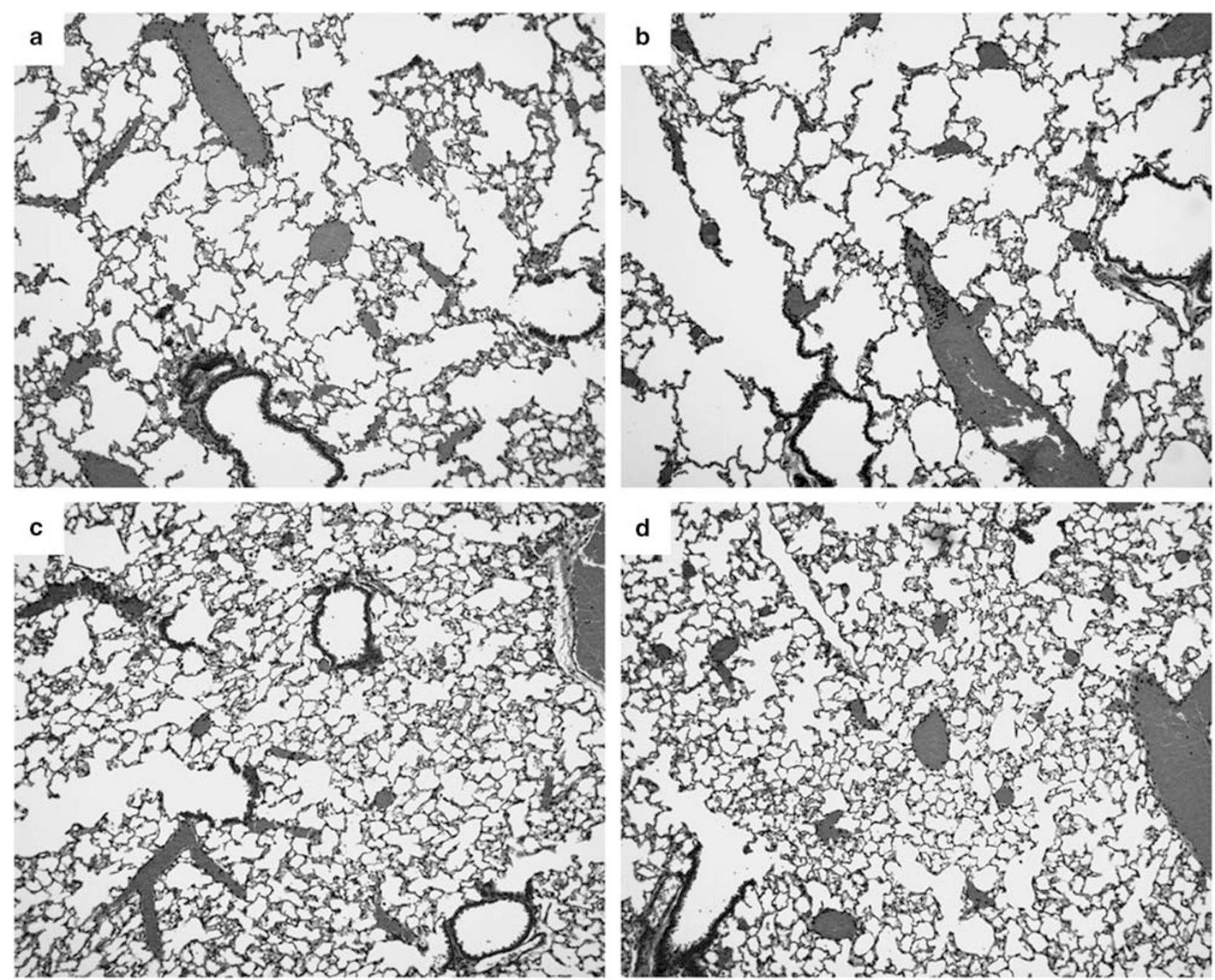

Figure 5 Histological analysis of Tsk1/ + and wild-type lungs. H\&E-stained sections of male Tsk1/ + (a and b) and wild-type (c and d) lungs at 12 weeks (a and $\mathbf{c}$ ) and 16 weeks $(\mathbf{b}$ and $\mathbf{d}$ ), pictures taken at $\times 10$ magnification.

The receptor Smads, Smad 2 and Smad 3, are phosphorylated in response to TGF $\beta$ and translocate to the nucleus. ${ }^{36}$ To confirm that the TGF $\beta$ pathway is not upregulated in the Tsk1/+ skin, we performed immunohistochemical analysis of Smad 2 phosphorylation levels in wild-type and Tsk1/+ skin sections taken from 6-week-old male mice. Figure 6c shows representative pictures of the staining levels. All epidermal and adnexal nuclei were phospho-Smad 2 positive in both wild-type and Tsk1/+, and a similar proportion of dermal nuclei were negative in both wild type and Tsk1/ + . Thus, there is no evidence that the TGF $\beta$ pathway is increased in the Tsk1/ + animals.

\section{Discussion}

The Tsk1/ + mouse has come to be used as model for human skin fibrosis and scleroderma. We have analyzed the Tsk1/ + mouse as a model system for testing the efficacy of potential antifibrotic agents in skin fibrosis. In order to measure changes in skin physiology over the course of a pharmacologic treatment, we measured the apparent thickness of the skin pinch at the scruff of the neck, and also the thickness of the ear pinna, because both of these have been shown to be increased in the Tsk1/+ mouse. ${ }^{19,37}$ Both of these measurements are easy to carry out, and demonstrated good correlation with phenotype. However, comparison of the skin pinch measurements with the histology of the dermis suggests that the augmented thickness of the skin pinch of the Tsk1/ + mouse is not due to increased thickness of the skin itself, but is caused by tethering of the dermis to the underlying fascia, as detailed in the original publication of this phenotype, ${ }^{18}$ and further characterized by Lemaire and colleagues. ${ }^{35}$ The increased variance in the measurement of the Tsk1/+ skin pinch compared to the wild-type mice is due to the fact that the tethering makes it hard to get a good pinch of skin to measure. 


\begin{tabular}{|c|c|c|c|c|c|c|}
\hline $\begin{array}{l}\text { a } \\
\text { gene name }\end{array}$ & $\begin{array}{l}\text { accession } \\
\text { number }\end{array}$ & $\begin{array}{l}\text { gene } \\
\text { symbol }\end{array}$ & Tsk1/+ & $\begin{array}{l}\text { wild } \\
\text { type }\end{array}$ & $\begin{array}{l}\text { Tsk1/+ } \\
\text { mean }\end{array}$ & $\begin{array}{l}\text { wild type } \\
\text { mean }\end{array}$ \\
\hline transforming growth factor, beta 1 & NM_011577 & Tgfb1 & & & 138 & 136 \\
\hline connective tissue growth factor & NM_010217 & Ctgf & & & 950 & 934 \\
\hline plasminogen activator inhibitor 1 & NM_008871 & Pai-1 & & & 153 & 188 \\
\hline thrombospondin 1 & $\mathrm{Al} 385532$ & Thbs1 & & & 315 & 250 \\
\hline cartilage oligomeric matrix protein & NM_016685 & Comp & & & 47 & 47 \\
\hline procollagen, type I, alpha 1 & U08020 & Col1a1 & & & 12845 & 12696 \\
\hline procollagen, type I, alpha 2 & BF227507 & Col1a2 & & & 8421 & 8273 \\
\hline procollagen, type IV, alpha 1 & BF158638 & Col4a1 & & & 1265 & 1372 \\
\hline procollagen, type III, alpha 1 & AW550625 & Col3a1 & & & 9264 & 8812 \\
\hline fibronectin 1 & BC004724 & Fn1 & & & 2228 & 2590 \\
\hline decorin & NM_007833 & Den & & & 7122 & 6839 \\
\hline biglycan & BC0̄19502 & Bgn & & & 1486 & 1559 \\
\hline
\end{tabular}
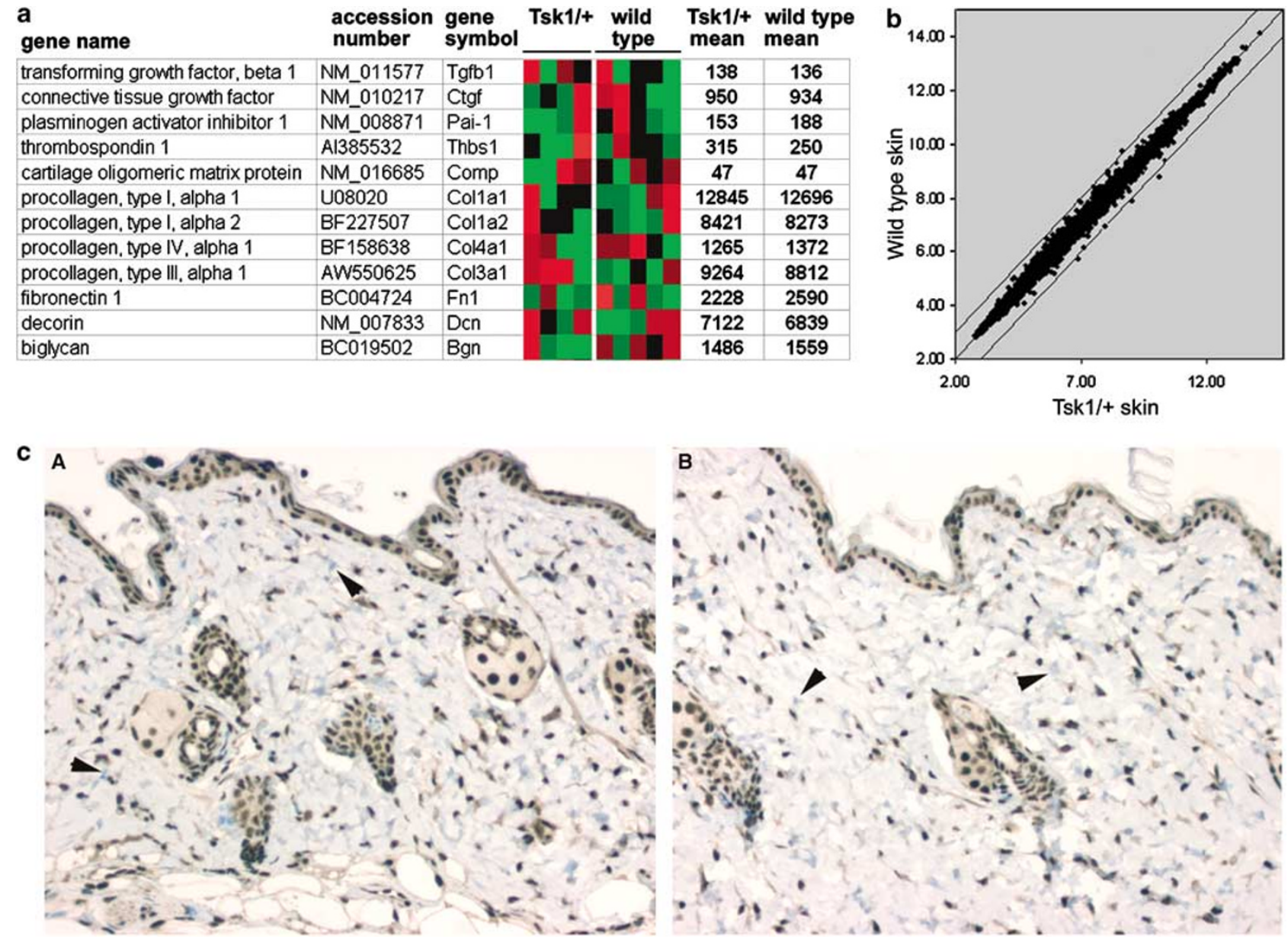

Figure 6 Transcript profiling of Tsk1/ + and wild-type skin. RNA was isolated from the skin taken from the scapular area of male Tsk1/ $+(n=4)$ and wild-type controls $(n=5)$ aged 6 weeks. (a) Comparison of expression levels of individual genes. For the heat map, red indicates higher expression and green lower expression, with the algorithm designed to compare each data point of a gene to the mean expression level of that gene. (b) Dot-blot comparing gene expression in wild-type and Tsk1/ + skin. (c) Immunohistochemical analysis of Smad 2 phosphorylation in wild-type and Tsk1/ + skin from 6-week-old mice, panel A Tsk1/+, panel B wild type. Arrowheads indicate examples of unstained nuclei. Total fibroblast nuclei were counted in two high magnification fields, and stained nuclei calculated as a percent of total, wild type $85.3 \pm 1.5 \%$, Tsk $1 /+83.0 \pm 5.7 \%$.

With a large sample size, it may be possible to use this measurement to assess the effects of gene deletions on the tethering phenotype, although it is unlikely to be useful in measuring effects of a pharmacologic agent. The ear pinna measurements showed that the Tsk1/+ ears were thinner than their wild-type counterparts, which is in direct contrast to previously published findings. ${ }^{37}$ This could be due to differences in the site of measurement; however, our finding that the tip of the ear pinna is thinner in the Tsk1/+ than in the wild-type mice supports the original diagnosis of this phenotype as one of tethering, rather than dermal hyperplasia, which would be expected to make the ear pinna thicker, not thinner as we see.

Next, we turned to measurements of dermal thickness and collagen content. We were surprised that in contrast to published work, we did not find dermal hyperplasia in this model, nor did we observe increased collagen content in the skin of Tsk1/ + mice either by histological staining or by quantitative real-time PCR for Col1a2 expression. In measuring the depth of the dermis in histological sections, we found considerable variation within and between sections, making it necessary to take multiple measurements of each section. When we had carried out these measurements on a large number of wild-type and Tsk1/ + samples, statistical analysis indicated that there was no difference between the two groups. In early experiments, we had observed significant differences in skin thickness between male and female mice (data not shown), and in all subsequent experiments we used only male mice. A recent publication showed increased collagen content in the skin of wild-type male mice compared to female, ${ }^{38}$ and this may suggest that some of the phenotypes previously ascribed to the Tsk1/ + mutation were due to sex or housing differences. 
Analysis of collagen gene expression by quantification of Picrosirius Red stain of skin sections, by real-time PCR analysis of Col1a2, and transcript profiling also did not reveal differences between wild-type and Tsk1/+ skin. While it is entirely possible that undetectable changes in expression can still result in increased collagen levels in the matrix as demonstrated in vitro by Lemaire and colleagues, ${ }^{39}$ we did not observe increased collagen by histological analysis of the dermis. Our results are in contrast with those of Denton et al, who reported increased activity of a col1a2-lacZ reporter in the Tsk1/+ mouse, and also demonstrated increased expression of Co1a1 and Col1a2 by Northern blot analysis. ${ }^{40}$ A potential explanation for the discrepancy might be that Tsk1/+ skin samples contain a larger amount of the attached subcutaneous tissue, leading to a larger percentage of fibroblasts in the sample. Although it has been shown that the levels of collagen transcription decrease with age, ${ }^{41}$ this occurs mainly in the first 2 weeks postnatally, with much smaller decreases thereafter and thus does not explain our results. We had planned to use the Tsk1/ + model to test the effects of antifibrotic therapies against skin fibrosis. In order to draw conclusions about effects of a pharmacologic agent, the difference between the wild-type and the Tsk1/ + mouse have to be significant and consistent to detect the effects of treatment. Based on the data that we have generated, any changes in dermal thickness and collagen content in the Tsk1/+ mouse are too subtle and variable to be used as a reliable end point. The tethering phenotype in the Tsk1/ + mouse may represent many aspects of scleroderma. Tethering is poorly represented in tissue sections due to the cleavage plane between the skin and subcutaneous tissues, but it is conceivable that antifibrotics could be assayed by their effects on tethering, either by a very reliable readout of the skin-fold thickness or by using sophisticated RNA retrieval from the subcutaneous fascia using laser capture.

It has been suggested that the Tsk1/ + phenotype is secondary to the activation of the TGF $\beta$ signaling axis. Crossing Tsk1/ + with mice lacking the TGF $\beta$ receptor, ${ }^{28}$ or with other knockouts that reduce the levels of TGF $\beta^{30}$ appears to abrogate the phenotype, and a polymorphism in the TGF $\beta$ promoter in Tsk1/+ mice increases expression of the gene. ${ }^{42}$ However, it has also been shown that TGF $\beta$ expression is not elevated in adult Tsk1/ + mouse skin. ${ }^{41}$ Fibrillin-1 has been proposed to have a role in controlling the biologically available levels of TGF $\beta$ through binding the LTBP, and it has recently been demonstrated that the emphysema seen in mice engineered to be deficient in fibrillin is caused by an increased activity of TGF $\beta .{ }^{11}$ The authors postulated that this is a direct effect of the decreased amount of fibrillin-1, which usually acts to sequester the latent complex rendering it biologically inaccessible. Thus, it would follow that a deficiency of fibrillin-
1 in the skin would have a similar effect, leading to increased activation of TGF $\beta$-responsive pathways. In support of this idea, it has been demonstrated that the microfibrils produced by Tsk1/ + fibroblasts are unstable, and the network is less abundant than those produced by wild-type cells. ${ }^{34}$ In addition, the microfibrils produced by scleroderma lesional fibroblasts also appear to be unstable. ${ }^{9}$ The hypothesis also requires that TGF $\beta$-induced signaling pathways be constitutively activated in the skin of Tsk1/+ mice. Again, this hypothesis is supported by the literature showing that crossing the Tsk1/ + mouse with lines that are deficient in TGF $\beta$ signaling appears to reduce the phenotype. In contrast, our transcript profiling data does not provide any evidence of increased activity of TGF $\beta$-induced signaling pathways, nor any increase in the expression of genes known to be involved in fibrosis. Interleukin-4 has also been implicated in the Tsk1/ + phenotype, ${ }^{30,43,44}$ and interestingly, deletion of the IL-4 gene is able to rescue homozygous Tsk1/ Tsk1 mice that usually die during embryonic development. $^{30,44}$ The authors suggest that the rescue of the Tsk1/Tsk1 homozygous mice in the absence of IL-4 signaling is through the secondary effect of decreased TGF $\beta$ signaling. However, the fact that our transcript profiling data shows no activation of the TGF $\beta$ pathway in the Tsk1/+ heterozygous mice suggests that the deletion of IL-4 has its effect through a TGF $\beta$-independent mechanism.

Based on the data presented here, we conclude that the skin phenotype of the Tsk1/ + mouse is primarily one of tethering and not of fibrosis, and that it is not caused by increased activity of TGF $\beta$ targeted pathways. Therefore, this model is not suitable for testing pharmacologic agents designed to target fibrosis, and it may not be as appropriate a model of scleroderma as has previously been proposed.

\section{References}

1 Sakai LY, Keene DR, Engvall E. Fibrillin, a new 350-kD glycoprotein is a component of microfibrils. J Cell Biol 1968;103:2499-2509.

2 Sakai LY, Keene DR. Fibrillin: monomers and microfibrils. Methods Enzymol 1994;245:29-52.

3 Wallis DD, Tan FK, Kessler R, et al. Fibrillin 1 abnormalities in dermal fibroblast cultures from firstdegree relatives of patients with systemic sclerosis (scleroderma). Arthritis Rheum 2004;50:329-331.

4 Arnett F, Howard R, Tan F, et al. Increased prevalence of systemic sclerosis in a Native American tribe in Oklahoma. Association with an Amerindian HLA haplotype. Arthritis Rheum 1996;39:1362-1370.

5 Tan FK, Stivers DN, Foster MW, et al. Association of microsatellite markers near the fibrillin 1 gene on human chromosome 15q with scleroderma in a Native American population. Arthritis Rheum 1998;41: 1729-1737. 
6 Fleischmajer R, Jacobs L, Schwartz E, et al. Extracellular microfibrils are increased in localized and systemic scleroderma skin. Lab Invest 1991;64: 791-798.

7 Tan FK, Arnett FC, Antohi S, et al. Autoantibodies to the extracellular matrix microfibrillar protein, fibrillin1 , in patients with scleroderma and other connective tissue diseases. J Immunol 1999;163:1066-1072.

8 Arnett FC, Tan FK, Uziel Y, et al. Autoantibodies to the extracellular matrix microfibrillar protein, fibrillin 1, in patients with localized scleroderma. Arthritis Rheum 1999;42:2656-2659.

9 Wallis DD, Tan FK, Kielty CM, et al. Abnormalities in fibrillin 1-containing microfibrils in dermal fibroblast cultures from patients with systemic sclerosis (scleroderma). Arthritis Rheum 2001;44:1855-1864.

10 Isogai Z, Ono RN, Ushiro S, et al. Latent transforming growth factor $\beta$-binding protein 1 interacts with fibrillin and is a microfibril-associated protein. J Biol Chem 2003;278:2750-2757.

11 Neptune ER, Frischmeyer PA, Arking DE, et al. Dysregulation of TGF- $\beta$ activation contributes to pathogenesis in Marfan syndrome. Nat Genet 2003;33: 407-411.

12 Unsold C, Hyytiainen M, Bruckner-Tuderman L, et al. Latent TGF-b binding protein LTBP-1 contains three potential extracellular matrix interacting domains. J Cell Sci 2000;114:187-197.

13 Saito S, Nishimura H, Brumeanu T-D, et al. Characterization of mutated protein encoded by partially duplicated fibrillin-1 gene in tight skin (TSK) mice. Mol Immunol 1999;36:169-176.

14 Trojanowska M. Molecular aspects of scleroderma. Front Biosci 2002;7:608-618.

15 Strehlow D, Korn JH. Biology of the scleroderma fibroblast. Curr Opin Rheum 1998;10:572-578.

16 Leask A, Abraham DJ. TGF-b signaling and the fibrotic response. FASEB J 2004;18:816-827.

17 Siracusa LD, McGrath R, Ma Q, et al. A tandem duplication within the fibrillin 1 gene is associated with the mouse tight skin mutation. Genome Res 1996;6:300-313.

18 Green MC, Sweet HO, Bunker LE. Tight-skin, a new mutation of the mouse causing excessive growth of connective tissue and skeleton. Am J Pathol 1976;82: 493-521.

19 Menton DN, Hess RA, Lichtenstein JR, et al. The structure and tensile properties of the skin of tight-skin (Tsk) mutant mice. J Invest Dermatol 1978; 70:4-10.

20 Menton DN, Hess RA. The ultrastructure of collagen in the dermis of tight-skin (Tsk) mutant mice. J Invest Dermatol 1980;74:139-147.

21 Jimenez SA, Millan A, Bashey RI. Scleroderma-like alterations in collagen metabolism occurring in the TSK (tight skin) mouse. Arthritis Rheum 1984;27: 180-185.

22 Hatakeyama A, Kasturi KN, Wolf I, et al. Correlation between the concentration of serum anti-topoisomerase I autoantibodies and histological and biochemical alterations in TSK mice. Cell Immunol 1996;167: 135-140.

23 Bocchieri MH, Jimenez SA. Animal models of fibrosis. Rheum Dis Clin N Am 1990;16:153-167.

24 Saito S, Kasturi K, Bona C. Genetic and immunologic features associated with scleroderma-like syndrome of TSK mice. Curr Rheumatol Rep 1999;1:34-37.
25 Pablos JL, Everett ET, Norris JS. The tight skin mouse: an animal model of systemic sclerosis. Clin Exp Rheumatol 2004;22:S81-S85.

26 Jimenez SA, Williams CJ, Myers JC, et al. Increased collagen biosynthesis and increased expression of type I and type III procollagen genes in the tight skin (TSK) mouse fibroblasts. J Biol Chem 1986;261:657-662.

27 Pines M, Domb A, Ohana M, et al. Reduction in dermal fibrosis in the tight-skin (Tsk) mouse after local application of halofuginone. Biochem Pharmacol 2001;62:1221-1227.

28 McGaha TL, Saito S, Phelps RG, et al. Lack of skin fibrosis in tight skin (TSK) mice with targeted mutation in the interleukin-4R alpha and transforming growth factor-beta genes. J Invest Dermatol 2001; 116:136-143.

29 McGaha TL, Phelps RG, Spiera H, et al. Halofuginone, an inhibitor of type-I collagen synthesis and skin sclerosis, blocks transforming-growth-factor- $\beta$-mediated smad3 activatin in fibroblasts. J Invest Dermatol 2002;118:461-470.

30 Kodera T, McGaha TL, Phelps R, et al. Disrupting the $I L-4$ gene rescues mice homozygous for the tight-skin mutation from embyronic death and diminishes TGF- $\beta$ production by fibroblasts. Proc Natl Acad Sci USA 2002;99:3800-3805.

31 McGaha TL, Bona C. Role of profibrogenic cytokines secreted by $\mathrm{T}$ cells in fibrotic processes in scleroderma. Autoimmun Rev 2002;1:174-181.

32 Levi-Schaffer F, Nagler A, Slavin S, et al. Inhibition of collagen synthesis and changes in skin morphology in murine graft-versus-host disease and tight skin mice: effect of halofuginone. J Invest Dermatol 1996;106: 84-88.

33 Phelps RG, Murai C, Saito S, et al. Effect of targeted mutation in collagen $\mathrm{V}$ alpha 2 gene on development of cutaneous hyperplasia in tight skin mice. Mol Med 1998;4:356-360.

34 Gayraud B, Keene DR, Sakai LY, et al. New insights into the assembly of extracellular microfibrils from the analysis of the fibrillin mutation in the tight skin mouse. J Cell Biol 2000;150:667-679.

35 Lemaire R, Korn JH, Schiemann WP, et al. Fibulin-2 and fibulin-5 alterations in Tsk mice associated with disorganized hypodermal elastic fibers and skin tethering. J Invest Dermatol 2004;123:1063-1069.

36 Moustakas A, Souchelnytskyi S, Heldin CH. Smad regulation in TGF-beta signal transduction. J Cell Sci 2001;114(Part 24):4359-4369.

37 Sundberg JP. The tight-skin (Tsk) mutation, chromosome 2. In: Sundberg JP (ed). Handbook of Mouse Mutations with Skin and Hair Abnormalities. CRC Press Inc.: Boca Raton, FL, 1994, pp 279-283.

38 Markova MS, Zeskand J, McEntee B, et al. A role for the androgen receptor in collagen content of the skin. J Invest Dermatol 2004;123:1052-1056.

39 Lemaire R, Farina G, Kissin E, et al. mutant fibrillin 1 from tight skin mice increases extracellular matrix incorporation of microfibril-associated glycoprotein 2 and type I collagen. Arthritis Rheum 2004;50: 915-926.

40 Denton CP, Zheng B, Shiwen X, et al. Activation of a fibroblast specific enhancer of the Pro $\alpha 2(\mathrm{I})$ collagen gene in tight-skin mice. Arthritis Rheum 2001;44: $712-722$.

41 Pablos JL, Everett ET, Harley R, et al. Transforming growth factor- $\beta 1$ and collagen gene expression during 
postnatal skin development and fibrosis in the tightskin mouse. Lab Invest 1995;72:670-678.

42 Zhu H, Bona C, McGaha TL. Polymorphisms of the TGF- $\beta 1$ promoter in tight skin (TSK) mice. Autoimmunity 2004;37:51-55.

43 Ong C, Wong C, Roberts CR, et al. Anti-IL-4 treatment prevents dermal collagen deposition in the tight-skin mouse model of scleroderma. Eur J Immunol 1998;28:2619-2629.

44 McGaha TL, Le M, Kodera T, et al. Molecular mechanisms of interleukin-4-induced up-regulation of type I collagen gene expression in murine fibroblasts. Arthritis Rheum 2003;48:2275-2284. 